\title{
Democratic Lagrangians for Nonlinear Electrodynamics
}

\author{
Zhirayr Avetisyan* \\ Department of Mathematics, University of California, Santa Barbara, California 93106-3080, USA \\ and Regional Mathematical Center of Southern Federal University, Rostov-on-Don 344090, Russia \\ Oleg Evnin $\oplus^{\dagger}$ \\ Department of Physics, Faculty of Science, Chulalongkorn University, Bangkok 10330, Thailand \\ and Theoretische Natuurkunde, Vrije Universiteit Brussel and International Solvay Institutes, Brussels 1050, Belgium \\ Karapet Mkrtchyan $\oplus^{\ddagger}$ \\ Theoretical Physics Group, Blackett Laboratory, Imperial College, London SW7 2AZ, United Kingdom
}

(Received 7 August 2021; accepted 8 November 2021; published 27 December 2021)

\begin{abstract}
We construct a Lagrangian for general nonlinear electrodynamics that features electric and magnetic potentials on equal footing. In the language of this Lagrangian, discrete and continuous electric-magnetic duality symmetries can be straightforwardly imposed, leading to a simple formulation for theories with the $\mathrm{SO}(2)$ duality invariance. When specialized to the conformally invariant case, our construction provides a manifestly duality-symmetric formulation of the recently discovered ModMax theory. We briefly comment on a natural generalization of this approach to $p$-forms in $2 p+2$ dimensions.
\end{abstract}

DOI: 10.1103/PhysRevLett.127.271601

Nonlinear electromagnetic theories with actions of the form

$$
S=\int \mathcal{L}(s, p) d^{4} x, \quad s \equiv \frac{1}{2} F_{\mu \nu} F^{\mu \nu}, \quad p \equiv \frac{1}{2} F_{\mu \nu} \star F^{\mu \nu}
$$

have surfaced historically in relation to topics as diverse as mitigating classical field divergences due to point charges [1-4], induced interactions of photons via coupling to matter [5-9], effective low-energy description of open string theory $[10,11]$, and construction of regular black holes [12-15]. Here, $F_{\mu \nu} \equiv \partial_{\mu} A_{\nu}-\partial_{\nu} A_{\mu}$ and the Hodge star is defined by $\star F_{\mu \nu}=\epsilon_{\mu \nu \sigma \rho} F^{\sigma \rho} / 2$, while most generally we view $\mathcal{L}$ as an arbitrary function.

There are many reasons one may want to recast (1) in a language where both the electric potential $A_{\mu}$ and its dual magnetic potential $B_{\mu}$ (satisfying $\star F_{\mu \nu} \equiv \partial_{\mu} B_{\nu}-\partial_{\nu} B_{\mu}$ in the free Maxwell theory) appear explicitly. For one thing, one may be interested in coupling this theory to both electrically and magnetically charged matter, as done for free theories in [16-19]. Furthermore, in many cases, such as the free Maxwell theory or the celebrated Born-Infeld

Published by the American Physical Society under the terms of the Creative Commons Attribution 4.0 International license. Further distribution of this work must maintain attribution to the author(s) and the published article's title, journal citation, and DOI. Funded by SCOAP.
(BI) theory $[1,10,11,20]$, the equations of motion are invariant under continuous rotations of $F$ into $\star F$ [2137], and it is desirable to have an action principle that manifests this symmetry [16,38-50].

The purpose of this Letter is to give a democratic formulation in terms of electric and magnetic potentials to a general theory of the form (1) and to explore the properties of this formulation for special cases of current interest. In our construction, we shall rely on the approach of [51,52], which, for free fields, provides a polynomial reformulation of the Pasti-Sorokin-Tonin (PST) theory [4042]. It turns out that this approach is well suited for including interactions, as we shall now demonstrate.

General formulation.-We start by recalling the formulation of [51,52] for free fields,

$$
\mathcal{L}_{\text {Maxwell }}=-\frac{1}{4} H_{\mu \nu}^{b} H^{b \mu \nu}+\frac{a(x)}{4} \epsilon_{b c} \varepsilon^{\mu \nu \lambda \rho} F_{\mu \nu}^{b} Q_{\lambda \rho}^{c},
$$

where $H_{\mu \nu}^{b} \equiv F_{\mu \nu}^{b}+a Q_{\mu \nu}^{b}, b=1,2, a(x)$ is an auxiliary scalar familiar from the PST theory, and

$$
F_{\mu \nu}^{b}=\partial_{\mu} A_{\nu}^{b}-\partial_{\nu} A_{\mu}^{b}, \quad Q_{\mu \nu}^{b}=\partial_{\mu} R_{\nu}^{b}-\partial_{\nu} R_{\mu}^{b} .
$$

Despite the large number of fields $\left(A^{b}, R^{b}, a\right)$, this Lagrangian describes a single propagating Maxwell field whose electric potential is $A^{1}$ and magnetic potential is $A^{2}$. To see this, we observe that (2) is invariant under the usual gradient shifts of $A^{b}$ and $R^{b}$ (the Lagrangian depends only on the corresponding field strengths) and has two additional symmetries, 


$$
\delta a=0, \quad \delta A_{\mu}^{b}=-a u^{b} \partial_{\mu} a, \quad \delta R_{\mu}^{b}=u^{b} \partial_{\mu} a,
$$

where $u^{b}(x)$ is an arbitrary doublet of scalar field parameters and another gauge symmetry that shifts $a(x)$ arbitrarily and correspondingly corrects the other fields. Further details can be found in [52]. As a consequence of these symmetries, any solution of the equations of motion can be gauged to

$$
R^{b}=0, \quad \star F_{\mu \nu}^{a}+\epsilon^{a b} F_{\mu \nu}^{b}=0,
$$

leaving a single propagating Maxwell field.

To include interactions in this formalism, we intend to deform the free action (2) in a way that maintains its gauge symmetries. To this end, we first note that $H_{\mu \nu}^{b}$ is invariant by itself under the transformations (4), while the second term in (2) changes by a total derivative. As a result, if we replace $H_{\mu \nu}^{b} H^{b \mu \nu}$ by an arbitrary scalar function of $H_{\mu \nu}^{b}$, the resulting interacting theory still automatically respects both gradient shifts of $A^{b}$ and $R^{b}$, and (4), which is crucial for the emergence of (5).

There are six functionally independent scalars one can build from $H_{\mu \nu}^{b}\left(U^{a b}=U^{b a}, V^{a b}=V^{b a}\right)$,

$$
U^{a b} \equiv \frac{1}{2} H_{\mu \nu}^{a} H^{b \mu \nu}, \quad V^{a b} \equiv \frac{1}{2} H_{\mu \nu}^{a} \star H^{b \mu \nu} .
$$

Our algorithm to construct a nonlinear generalization of (2) is then to start with

$$
\mathcal{L}=a \epsilon_{b c} F^{b} \wedge Q^{c}+f\left(U,{ }^{11} U,{ }^{12} U,{ }^{22} V,{ }^{11} V,{ }^{12} V^{22}\right),
$$

and constrain $f$ by the requirement that there is an extra symmetry shifting $a(x)$ arbitrarily, so that it is a pure gauge degree of freedom, or equivalently [52], that the equations of motion for $A^{b}$ and $R^{b}$,

$d\left[\left(f_{b c}^{U}+f_{c b}^{U}\right) \star H^{c}-\left(f_{b c}^{V}+f_{c b}^{V}\right) H^{c}+a \epsilon_{b c} Q^{c}\right]=0$,

$d\left[a\left\{\left(f_{b c}^{U}+f_{c b}^{U}\right) \star H^{c}-\left(f_{b c}^{V}+f_{c b}^{V}\right) H^{c}-\epsilon_{b c} F^{c}\right\}\right]=0$,

where $f_{a b}^{U} \equiv \partial f / \partial U_{a b}, f_{a b}^{V} \equiv \partial f / \partial V_{a b}\left(f_{21}^{U} \equiv 0 \equiv f_{21}^{V}\right)$, imply the equation of motion for $a(x)$,

$$
Q^{b} \wedge K_{b}=0,
$$

with

$$
K_{a} \equiv\left(f_{a b}^{U}+f_{b a}^{U}\right) \star H^{b}-\left(f_{a b}^{V}+f_{b a}^{V}\right) H^{b}-\epsilon_{a b} H^{b} .
$$

For that, we first note that, multiplying (8) by $a(x)$ and subtracting it from (9), one gets (we use the differential form notation following the derivations of [52])

$$
d a \wedge K_{b}=0 .
$$

There is a natural way to ensure that (12) implies (10) in a manner analogous to the free theory [52]. Indeed, if

$$
K_{a} \pm \epsilon_{a b} \star K_{b} \equiv 0,
$$

then (12) implies $K_{b}=0$ by elementary differential form algebra. Hence, (10) is satisfied whenever (8) and (9) are satisfied [53].

One can translate (13) to the following condition on $f$ :

$$
\pm \delta^{a c}\left(f_{c b}^{U}+f_{b c}^{U}\right)-\epsilon^{a c}\left(f_{c b}^{V}+f_{b c}^{V}\right)+\delta_{b}^{a}=0 .
$$

These linear partial differential equations (PDEs) are solved in full generality by

$$
\begin{gathered}
f(U, V)=\mp \frac{1}{2} U_{a a}+g\left(\lambda_{1}, \lambda_{2}\right), \\
\lambda_{1}= \pm U_{12}-\frac{1}{2}\left(V_{11}-V_{22}\right), \\
\lambda_{2}=V_{12} \pm \frac{1}{2}\left(U_{11}-U_{22}\right),
\end{gathered}
$$

where $g\left(\lambda_{1}, \lambda_{2}\right)$ is an arbitrary function (we will henceforth use the upper signs only). The Lagrangian is then

$$
\mathcal{L}=\mathcal{L}_{\text {Maxwell }}+g\left(\lambda_{1}, \lambda_{2}\right),
$$

where $\lambda_{1,2}$ can be read off (16), (17), and (6). This Lagrangian respects a gauge symmetry that shifts $a$,

$\delta a=\varphi(x), \quad \delta A_{\mu}^{b}=-a \delta R_{\mu}^{b}=\frac{\varphi}{(\partial a)^{2}} a \partial^{\nu} a\left(Q_{\nu \mu}^{b}-\epsilon^{b c} \star Q_{\nu \mu}^{c}\right)$.

The gauge transformation rules do not depend on the function $g\left(\lambda_{1}, \lambda_{2}\right)$ and, in particular, are the same as in the free case [52]; $\lambda_{1,2}$ are invariant under (4) and (19). The Lagrangian (18) contains a single arbitrary function of two variables, as does (1). We shall proceed to show its relation to the single-field formulation (1) after discussing the surprisingly simple way additional electric-magnetic symmetries can be imposed on (18).

Duality symmetry.-Under the discrete $Z_{4}$ interchange of electric and magnetic degrees of freedom, $H^{1} \rightarrow H^{2}$, $H^{2} \rightarrow-H^{1}$, and hence $\lambda_{1} \rightarrow-\lambda_{1}, \lambda_{2} \rightarrow-\lambda_{2}$. Therefore, theories with such discreet duality symmetry are described by $g\left(\lambda_{1}, \lambda_{2}\right)$ satisfying

$$
g\left(-\lambda_{1},-\lambda_{2}\right)=g\left(\lambda_{1}, \lambda_{2}\right) .
$$

Next, one may ask for the full $\mathrm{SO}(2)$ duality symmetry with respect to rotating $A^{b}$ and $R^{b}$ in the $b$-plane. Under such a rotation by an angle $\alpha$, the pair $\left(\lambda_{1}, \lambda_{2}\right)$ simply rotates as a vector by an angle $2 \alpha$; therefore, only the radial part is 
invariant. Hence, the SO(2)-invariant theories are encoded in full generality by

$$
g\left(\lambda_{1}, \lambda_{2}\right)=h(w), \quad w=\sqrt{\lambda_{1}^{2}+\lambda_{2}^{2}},
$$

and their Lagrangian is given by

$$
\mathcal{L}=\mathcal{L}_{\text {Maxwell }}+h(w) .
$$

One can show that

$$
w=\sqrt{-\operatorname{det} \mathcal{H}},
$$

where $\mathcal{H}^{a b} \equiv\left(\star H_{\mu \nu}^{a}-\epsilon^{a c} H_{\mu \nu}^{c}\right)\left(\star H^{b \mu \nu}-\epsilon^{b d} H^{d \mu \nu}\right) / 2$.

Conformal invariance.-Another symmetry one may require from (1) is conformal invariance [34-36], which can be expressed by a condition of the form

$$
U^{a b} f_{a b}^{U}+V^{a b} f_{a b}^{V}=f .
$$

Then, $g\left(\lambda_{1}, \lambda_{2}\right)$ is a homogeneous function of degree one,

$$
g=\lambda_{1} \tilde{g}\left(\lambda_{1} / \lambda_{2}\right)
$$

where $\tilde{g}(x)$ is an arbitrary function.

If we require both $\mathrm{SO}(2)$ symmetry and conformal invariance, (22) reduces to

$$
\mathcal{L}=-\frac{1}{2} H^{b} \wedge \star H^{b}+a \epsilon_{b c} F^{b} \wedge Q^{c}+\delta w,
$$

where $\delta$ is an arbitrary real number, and $w$ is given by (23). As we shall show below, this construction provides an explicit duality-symmetric formulation of the ModMax theory recently introduced in [34] (see also [35-37]).

Analysis of the equations of motion.-As explained under (13), Eqs. (8) and (9) imply

$$
K_{b}=0
$$

for theories of the form (18). Plugging (27) into (8), we deduce

$$
d a \wedge d R^{b}=0,
$$

exactly the same equation as for the free field case [52]. This, in turn, implies that the fields $R^{b}$ can be gauge transformed to zero (see [52] for details),

$$
R^{b}=0, \quad H^{b}=F^{b} .
$$

Note that the two equations (27) are Hodge-dual to each other due to (13) and can hence be expressed as a single equation $\left(g_{1} \equiv \partial g / \partial \lambda_{1}, g_{2} \equiv \partial g / \partial \lambda_{2}\right)$,

$$
\star F^{1}+F^{2}=g_{2}\left(\star F^{1}-F^{2}\right)-g_{1} \star\left(\star F^{1}-F^{2}\right),
$$

which includes the free case (5) given by $g\left(\lambda_{1}, \lambda_{2}\right)=0$. Taking into account that (with $R^{b}=0$ )

$$
\lambda_{1}=\frac{1}{2} G_{\mu \nu} \star G^{\mu \nu}, \quad \lambda_{2}=-\frac{1}{2} G_{\mu \nu} G^{\mu \nu},
$$

where $G_{\mu \nu} \equiv \star F_{\mu \nu}^{1}-F_{\mu \nu}^{2}, g\left(\lambda_{1}, \lambda_{2}\right)$ is expressed through $G$, while the right-hand side of (30) is equal to $-\partial g / \partial G$. Therefore, (30) is an expression for $\star F^{1}+F^{2}$ in terms of $\star F^{1}-F^{2}$. This means that, similar to the free field case, there is only one independent field strength, while all the auxiliary fields have been gauged away. Hence, we are really dealing with a theory of one dynamical gauge field, as intended.

Relation to the conventional single-field formulation.The two formulations of nonlinear electrodynamics, given by (1) and (18), are related by nonlinear algebraic equations involving derivatives of the corresponding Lagrangians. We now proceed to establish this relation. If one solves (30) for $F_{1}$, by Lorentz invariance, the resulting expression must be of the form (see, e.g., [33])

$$
F^{1}=\alpha(s, p) F^{2}+\beta(s, p) \star F^{2},
$$

where $s$ and $p$ are the two independent invariants of $F^{2}$,

$$
s=\frac{1}{2} F_{\mu \nu}^{2} F^{2 \mu \nu}, \quad p=\frac{1}{2} F_{\mu \nu}^{2} \star F^{2 \mu \nu},
$$

and $\alpha$ and $\beta$ depend on the specific form of $g$. If we apply the exterior derivative operator to (32), the left-hand side vanishes, and $F^{1}$ disappears from the equation. What remains is the equation of motion for the theory (1), where $F$ is identified with $F^{2}$ and

$$
\alpha(s, p)=-\frac{\partial \mathcal{L}}{\partial p}, \quad \beta(s, p)=\frac{\partial \mathcal{L}}{\partial s} .
$$

Thus, our democratic formulation reproduces dynamically the theory (1) for a single electromagnetic field with selfinteractions.

One can recast (32) as the following equations in terms of the invariants:

$$
g_{1}=\frac{2 \alpha}{\alpha^{2}+(\beta+1)^{2}}, \quad g_{2}=\frac{\alpha^{2}+\beta^{2}-1}{\alpha^{2}+(\beta+1)^{2}} .
$$

Here, $g$ is specified as a function of $\lambda_{1}$ and $\lambda_{2}$. The latter can be extracted from (32), (16), and (17) as

$$
\begin{aligned}
& \lambda_{1}=2 \alpha(1+\beta) s-\left[\alpha^{2}-(1+\beta)^{2}\right] p, \\
& \lambda_{2}=\left[\alpha^{2}-(1+\beta)^{2}\right] s+2 \alpha(1+\beta) p,
\end{aligned}
$$


so that

$$
w \equiv \sqrt{\lambda_{1}^{2}+\lambda_{2}^{2}}=\left(\alpha^{2}+(\beta+1)^{2}\right) \sqrt{s^{2}+p^{2}} .
$$

For any concrete $g\left(\lambda_{1}, \lambda_{2}\right)$, (35) provides a $2 \times 2$ system of nonlinear algebraic equations for $\alpha$ and $\beta$ as functions of $s$ and $p$. Given a solution of this system, one can reduce the equations of motion of the democratic theory to the singlefield form (32). Conversely, to recast a given single-field theory of the form (1) in terms of the democratic formulation (18), one needs to obtain $\alpha$ and $\beta$ from (34), then $\lambda_{1}$ and $\lambda_{2}$ from (36), and then $g$ from (35).

Single-field formulation for duality-symmetric theories.-There is little one can say in general about solutions to (35) and (36), but extra structures emerge for special classes of theories. For the SO(2)-invariant case of (22), one gets

$\frac{\lambda_{1}}{w} h^{\prime}=\frac{2 \alpha}{\alpha^{2}+(\beta+1)^{2}}, \quad \frac{\lambda_{2}}{w} h^{\prime}=\frac{\alpha^{2}+\beta^{2}-1}{\alpha^{2}+(\beta+1)^{2}}$.

Eliminating $h^{\prime}$ gives $\lambda_{1}\left(\alpha^{2}+\beta^{2}-1\right)=2 \alpha \lambda_{2}$, and then

$$
\beta^{2}+\frac{2 s}{p} \alpha \beta-\alpha^{2}=1
$$

which is, in view of (34), exactly the same as the general $\mathrm{SO}(2)$-invariance condition in the single-field formalism $[21,23,28,31,35,36,46]$. The remaining equation can then be written as

$$
\left.[\alpha s+(\beta+1) p] h^{\prime}\right|_{w=\sqrt{s^{2}+p^{2}}\left[\alpha^{2}+(\beta+1)^{2}\right]}=\alpha \sqrt{s^{2}+p^{2}} .
$$

If one is moving from the single-field formalism to the democratic one for $\mathrm{SO}(2)$-invariant theories, (39) is satisfied from the start, and (40) is what must be solved to reconstruct $h(w)$.

The ModMax theory.-For the conformally invariant case (26), Eq. (40) becomes linear, and it can be solved together with (39),

$$
\begin{gathered}
\alpha(s, p)=-\sinh \gamma \frac{p}{\sqrt{p^{2}+s^{2}}}, \\
\beta(s, p)=\sinh \gamma \frac{s}{\sqrt{p^{2}+s^{2}}}-\cosh \gamma .
\end{gathered}
$$

With these functions, (32) reproduces the equations of motion of the ModMax theory introduced in [34] and defined by the Lagrangian

$$
L(s, p)=-\cosh \gamma s+\sinh \gamma \sqrt{s^{2}+p^{2}} .
$$

Thus, with the identification

$$
\delta=\operatorname{coth} \frac{\gamma}{2}
$$

a democratic description of the ModMax theory is provided by (26). This solution corresponds to $\delta>1(\gamma>0)$ or $\delta<$ $-1(\gamma<0)$. For the region $-1<\delta<1$, which can be given as $\delta=\tanh (\gamma / 2)$, we get the same action (43), but with an overall minus sign. This is what happens if we replace $F_{\mu \nu} \rightarrow \star F_{\mu \nu}, \gamma \rightarrow-\gamma$ in the Lagrangian (43), so it is the ModMax theory written in terms of the dual magnetic potential. The case $\delta=1$ [54] corresponds to the Białynicki-Birula electrodynamics [55,56].

Other duality-invariant theories.-While we have outlined the general way to connect the single-field and democratic formulations, it involves solving nonlinear algebraic equations, which is only possible explicitly in special cases. Furthermore, the functions appearing in the democratic Lagrangian may be complicated for known simple single-field theories and vice versa.

We demonstrate here the conversion procedure for the generalized BI theory $[34,36]$,

$L_{\mathrm{GBI}}=\sqrt{\mathrm{UV}}-T, \quad U \equiv 2 u+e^{\gamma} T, \quad V \equiv-2 v+e^{-\gamma} T$,

where $u \equiv\left(s+\sqrt{p^{2}+s^{2}}\right) / 2, v \equiv\left(-s+\sqrt{p^{2}+s^{2}}\right) / 2$, and $T$ is an arbitrary constant (related to the string tension in [10]). The BI theory corresponds to $\gamma=0$. From (38),

$$
h^{\prime}=\frac{\sqrt{U}-\sqrt{V}}{\sqrt{U}+\sqrt{V}} .
$$

From (36),

$$
w=\frac{T}{2}\left(e^{-\gamma} \frac{U}{V}-e^{\gamma}\right)\left(1+\sqrt{\frac{V}{U}}\right)^{2} .
$$

Introducing $e^{\lambda} \equiv \sqrt{V / U}$, one can multiply (46) by $\partial w / \partial \lambda$ and integrate to obtain

$$
\begin{gathered}
h(\lambda)=4 T \sinh ^{2} \frac{\lambda}{2} \cosh (\lambda+\gamma), \\
w(\lambda)=-4 T \cosh ^{2} \frac{\lambda}{2} \sinh (\lambda+\gamma) .
\end{gathered}
$$

These formulas provide an implicit definition for $h(w)$ corresponding to the generalized BI theory in the democratic formulation (22).

Conclusions. - We have provided a democratic formulation (18) for the nonlinear electrodynamics (1) that explicitly features both electric and magnetic gauge potentials. It includes two auxiliary gauge fields and an auxiliary scalar, all of which are pure gauge degrees of freedom. The propagating degrees of freedom are those of (1). 
The $\mathrm{SO}(2)$ duality invariance is expressed in this formalism by the strikingly simple condition (21), which takes the place of the nonlinear PDE given by (39) and (34) responsible for the same property in the single-field formalism (this PDE is sometimes referred to [31,46] as the Courant-Hilbert equation after the classic treatise [57]). This makes it easy to specify arbitrary $\mathrm{SO}(2)$-invariant interactions in (22), without any need to satisfy additional constraints. In particular, polynomial interactions can be straightforwardly introduced by choosing $h(w)$ in (22) as a polynomial in $w^{2}$. If conformal symmetry is imposed in addition to the $\mathrm{SO}(2)$ invariance, the theory further simplifies to (26), which is a democratic formulation of the ModMax theory introduced in [34].

Our formulation has been developed as a nonlinear extension of the approach employed for free fields in [51,52]. In the free field context, this approach is closely related to the PST formulation [40-42], which is recovered by integrating out the auxiliary form fields. The relation is less obvious for the interacting theories discussed here since the equations of motion are no longer linear. If the auxiliary gauge fields can be successfully integrated out, a PST-like formulation of nonlinear electrodynamics will be produced.

Our approach to constructing the Lagrangian (18) has been rather systematic in that we started with a simple Lagrangian ansatz that automatically respects all the necessary gauge symmetries except for the symmetry that shifts the auxiliary scalar. Then, enforcing this last symmetry fixed the form of the Lagrangian. An advantage of the resulting theory (18) is that all the gauge symmetries are realized in a universal manner, independent of the form of interactions [58].

The approach adopted here naturally lends itself to generalizations to higher form field interactions, and it would be interesting to explore them. An educated guess is that for $(2 k-1)$-form fields in $4 k$ dimensions, the most general nonlinear democratic Lagrangian is

$$
\mathcal{L}=\mathcal{L}_{\text {free }}+g\left(\lambda_{i}\right)
$$

where the first term is the free Lagrangian from [52] and the second term is an arbitrary function of all independent Lorentz scalars $\lambda_{i}$ built out of the tensor $\star H^{a}-\epsilon^{a b} H^{b}$. The equations of motion will imply a deformed twisted selfduality relation that expresses, as in $(30), \star F^{a}+\epsilon^{a b} F^{b}$ as a function of the opposite chirality combination $\star F^{a}-\epsilon^{a b} F^{b}$. The $\mathrm{SO}(2)$ symmetry condition will further constrain $g$ to depend on a specific set of combinations of $\lambda_{i}$. Similarly, for the chiral $2 k$-forms in $4 k+2$ dimensions, a natural guess is of the same form (49), with the free part given as in [51,52], while now $\lambda_{i}$ are all independent Lorentz scalars built out of the tensor $\star H-H$. These structures will be explored in more detail in future works.
The authors are grateful to Euihun Joung and Arkady Tseytlin for helpful discussions. O. E. has been supported by the CUniverse research promotion project (CUAASC) at Chulalongkorn University. K. M. is supported by the European Union's Horizon 2020 Research and Innovation Programme under the Marie Skłodowska-Curie Grant No. 844265. Z. A. acknowledges the support of the Ministry of Education and Science of Russia, Agreement No. 075-02-2021-1386.

*z.avetisyan@math.ucsb.edu

†oleg.evnin@gmail.com

*k.mkrtchyan@imperial.ac.uk

[1] M. Born and L. Infeld, Foundations of the new field theory, Proc. R. Soc. A 144, 425 (1934).

[2] J. Plebański, Lectures on Non-Linear Electrodynamics (Nordita, Copenhagen, 1970).

[3] A. A. Tseytlin, On singularities of spherically symmetric backgrounds in string theory, Phys. Lett. B 363, 223 (1995).

[4] O. Pasarin and A. A. Tseytlin, Generalised Schwarzschild metric from double copy of point-like charge solution in Born-Infeld theory, Phys. Lett. B 807, 135594 (2020).

[5] W. Heisenberg and H. Euler, Folgerungen aus der Diracschen Theorie des Positrons, Z. Phys. 98, 714 (1936).

[6] Z. Białynicka-Birula and I. Białynicki-Birula, Nonlinear effects in quantum electrodynamics: Photon propagation and photon splitting in an external field, Phys. Rev. D 2, 2341 (1970).

[7] S. L. Adler, Photon splitting and photon dispersion in a strong magnetic field, Ann. Phys. (N.Y.) 67, 599 (1971).

[8] I. T. Drummond and S. J. Hathrell, QED vacuum polarization in a background gravitational field and its effect on the velocity of photons, Phys. Rev. D 22, 343 (1980).

[9] G. V. Dunne, Heisenberg-Euler effective Lagrangians: Basics and extensions, in From Fields to Strings: Circumnavigating Theoretical Physics (World Scientific, Singapore, 2005).

[10] E. S. Fradkin and A. A. Tseytlin, Nonlinear electrodynamics from quantized strings, Phys. Lett. 163B, 123 (1985).

[11] A. A. Tseytlin, Born-Infeld action, supersymmetry and string theory, in The Many Faces of the Superworld (World Scientific, Singapore, 2000).

[12] E. Ayón-Beato and A. García, Regular Black Hole in General Relativity Coupled to Nonlinear Electrodynamics, Phys. Rev. Lett. 80, 5056 (1998).

[13] K. A. Bronnikov, Regular magnetic black holes and monopoles from nonlinear electrodynamics, Phys. Rev. D 63, 044005 (2001).

[14] I. Dymnikova, Regular electrically charged structures in nonlinear electrodynamics coupled to general relativity, Classical Quantum Gravity 21, 4417 (2004).

[15] L. Balart and E. C. Vagenas, Regular black holes with a nonlinear electrodynamics source, Phys. Rev. D 90, 124045 (2014).

[16] D. Zwanziger, Local Lagrangian quantum field theory of electric and magnetic charges, Phys. Rev. D 3, 880 (1971).

[17] M. Blagojević and P. Senjanović, The quantum field theory of electric and magnetic charge, Phys. Rep. 157, 233 (1988). 
[18] K. Lechner and P. A. Marchetti, Duality invariant quantum field theories of charges and monopoles, Nucl. Phys. B569, 529 (2000).

[19] M. Henneaux and C. Troessaert, A note on electric-magnetic duality and soft charges, J. High Energy Phys. 06 (2020) 081.

[20] M. Abou Zeid and C. M. Hull, Geometric actions for D-branes and M-branes, Phys. Lett. B 428, 277 (1998).

[21] M. K. Gaillard and B. Zumino, Duality rotations for interacting fields, Nucl. Phys. B193, 221 (1981).

[22] I. Białynicki-Birula, Nonlinear electrodynamics: Variations on a theme by Born and Infeld, in Quantum Theory of Particles and Fields: Birthday Volume Dedicated to Jan Łopuszański (World Scientific, Singapore, 1983), pp. 31-48.

[23] G. W. Gibbons and D. A. Rasheed, Electric-magnetic duality rotations in nonlinear electrodynamics, Nucl. Phys. B454, 185 (1995).

[24] A. A. Tseytlin, Self-duality of Born-Infeld action and Dirichlet three-brane of type IIB superstring theory, Nucl. Phys. B469, 51 (1996).

[25] M. Perry and J. H. Schwarz, Interacting chiral gauge fields in six-dimensions and Born-Infeld theory, Nucl. Phys. B489, 47 (1997).

[26] M. K. Gaillard and B. Zumino, Self-duality in nonlinear electromagnetism, Lect. Notes Phys. 509, 121 (1998).

[27] M. K. Gaillard and B. Zumino, Nonlinear electromagnetic self-duality and Legendre transformations, arXiv:hep-th/ 9712103.

[28] M. Hatsuda, K. Kamimura, and S. Sekiya, Electric magnetic duality invariant Lagrangians, Nucl. Phys. B561, 341 (1999).

[29] S. M. Kuzenko and S. Theisen, Supersymmetric duality rotations, J. High Energy Phys. 03 (2000) 034.

[30] S. M. Kuzenko and S. Theisen, Nonlinear self-duality and supersymmetry, Fortschr. Phys. 49, 273 (2001).

[31] E. A. Ivanov and B. M. Zupnik, New approach to nonlinear electrodynamics: Dualities as symmetries of interaction, Phys. At. Nucl. 67, 2188 (2004).

[32] C. Bunster and M. Henneaux, Duality Invariance Implies Poincaré Invariance, Phys. Rev. Lett. 110, 011603 (2013).

[33] G. Buratti, K. Lechner, and L. Melotti, Duality invariant self-interactions of Abelian $p$-forms in arbitrary dimensions, J. High Energy Phys. 09 (2019) 022.

[34] I. Bandos, K. Lechner, D. Sorokin, and P. K. Townsend, A non-linear duality-invariant conformal extension of Maxwell's equations, Phys. Rev. D 102, 121703(R) (2020).

[35] B. P. Kosyakov, Nonlinear electrodynamics with the maximum allowable symmetries, Phys. Lett. B 810, 135840 (2020).

[36] I. Bandos, K. Lechner, D. Sorokin, and P. K. Townsend, On $p$-form gauge theories and their conformal limits, J. High Energy Phys. 03 (2021) 022.

[37] S. M. Kuzenko, Superconformal duality-invariant models and $\mathcal{N}=4$ SYM effective action, arXiv:2106.07173.

[38] M. Henneaux and C. Teitelboim, Dynamics of chiral (selfdual) p-forms, Phys. Lett. B 206, 650 (1988).

[39] J. H. Schwarz and A. Sen, Duality symmetric actions, Nucl. Phys. B411, 35 (1994).

[40] P. Pasti, D. P. Sorokin, and M. Tonin, Note on manifest Lorentz and general coordinate invariance in duality symmetric models, Phys. Lett. B 352, 59 (1995).
[41] P. Pasti, D. P. Sorokin, and M. Tonin, Duality symmetric actions with manifest space-time symmetries, Phys. Rev. D 52, R4277 (1995).

[42] P. Pasti, D. P. Sorokin, and M. Tonin, On Lorentz invariant actions for chiral $p$-forms, Phys. Rev. D 55, 6292 (1997).

[43] D. Berman, $S L(2, Z)$ duality of Born-Infeld theory from non-linear self-dual electrodynamics in 6 dimensions, Phys. Lett. B 409, 153 (1997).

[44] A. Nurmagambetov, Duality symmetric three-brane and its coupling to type IIB supergravity, Phys. Lett. B 436, 289 (1998).

[45] M. Roček and A. A. Tseytlin, Partial breaking of global $D=4$ supersymmetry, constrained superfields, and threebrane actions, Phys. Rev. D 59, 106001 (1999).

[46] X. Bekaert and S. Cucu, Deformations of duality symmetric theories, Nucl. Phys. B610, 433 (2001).

[47] C. Bunster and M. Henneaux, The action for twisted selfduality, Phys. Rev. D 83, 125015 (2011).

[48] C. Bunster and M. Henneaux, $\operatorname{Sp}(2 n, R)$ electric-magnetic duality as off-shell symmetry of interacting electromagnetic and scalar fields, Proc. Sci. HRMS2010 (2010) 028 [arXiv:1101.6064].

[49] P. Pasti, D. Sorokin, and M. Tonin, Covariant actions for models with non-linear twisted self-duality, Phys. Rev. D 86, 045013 (2012).

[50] E. A. Ivanov, A. J. Nurmagambetov, and B. M. Zupnik, Unifying the PST and the auxiliary tensor field formulations of 4D self-duality, Phys. Lett. B 731, 298 (2014).

[51] K. Mkrtchyan, On covariant actions for chiral $p$-forms, J. High Energy Phys. 12 (2019) 076.

[52] S. Bansal, O. Evnin, and K. Mkrtchyan, Polynomial dualitysymmetric Lagrangians for free $p$-forms, Eur. Phys. J. C 81, 257 (2021).

[53] We do not have a proof that (13) is the only way to ensure the gauge symmetry that shifts $a$, but it is satisfactory in that it allows us to construct a sufficiently large class of theories.

[54] For $\delta=1$, one cannot resolve (30) into (32). Rather, (30) implies that $\mathbf{V}=0$, $\operatorname{det} \mathbf{U}=0$, where $\mathbf{V}$ and $\mathbf{U}$ are the $2 \times 2$ matrices defined by (6) with $H^{b} \rightarrow F^{b}$. These equations manifest enhanced $S L(2, R)$ symmetry. One can then always apply a duality rotation, so that $U_{11}=0=V_{a b}$. Thus, the electric field strength satisfies $s=0=p$ in the notation of (1), known as the equations of motion of the BiałynickiBirula electrodynamics [55,56] (see also [36]). This theory does not admit a single-potential Lagrangian formulation, while it arises naturally from the democratic Lagrangian we consider here, highlighting the power of our formalism.

[55] I. Białynicki-Birula, Field theory of photon dust, Acta Phys. Pol. B 23, 553 (1992).

[56] D. Chruściński, Strong field limit of the Born-Infeld $p$-form electrodynamics, Phys. Rev. D 62, 105007 (2000).

[57] R. Courant and D. Hilbert, Methods of Mathematical Physics (Wiley \& Sons, New York, 1989), Vol. 2, p. 91.

[58] Even though this approach can access generic theories of the form (1), it would be interesting to check if there also exist theories that deform the symmetries (4) and (19) of the free theory. 